\title{
Dynamic analysis of bending-torsion coupled composite beams using the Flexibility Influence Function Method
}

\author{
C. Santiuste, S. Sánchez-Sáez, E. Barbero* \\ Department of Continuum Mechanics and Structural Analysis, University Carlos III of Madrid, Avda. de la Universidad 30, 28911 Leganés, Madrid, Spain
}

\section{A R T I C L E I N F O}

Keywords:

Bending-torsion coupling

Flexibility Influence Function Method

First-order Shear-Deformation Theory

Composite laminates

Beam models

\begin{abstract}
A B S T R A C T
In this work the dynamic behaviour of symmetrical laminated beams was studied, taking into account the effect of bending torsion coupling by a one dimensional model. This model includes the influence of the shear force and rotatory inertia. To solve the equations of motion, the Flexibility Influence Function Method (FIFM) was used. The dynamic displacements (deflection, bending rotation, and torsional rotation) were calculated for a beam in which the deflection and torsional rotation were restricted at its both ends, allowing the bending rotation. The accuracy of this method was determined by using a Three Dimensional Finite Element Method (FEM3D) model to compare the dynamic displacements. The need was shown to incorporate coupling in the one dimensional model in order to calculate the dynamic deflection and bending rotation of a composite beam.
\end{abstract}

\section{Introduction}

The design of structural elements such as beams manufactured with composite laminates requires the use of models that consider the specific characteristics of these materials, such as their high anisotropy, their sensitivity to interlaminar shear stress, and possible elastic coupling effects (bending torsion, extension torsion, extension bending, etc.) [1]. In addition, these types of elements may be subjected to dynamic loads, which require effects of inertia to be introduced into the models in order to ensure proper design.

The existence of coupling phenomena increases the complexity of formulating the equations of motion and of the procedures to resolve the problem [2]. The coupling can be controlled by laminate parameters (ply orientation and stacking sequence), modifying the global response of the structure [3]. Thus, for example, in helicopter rotors, it is possible to control the aeroelastic response of the blades by the elastic coupling; the vibrations of the rotor could be reduced and the aerodynamic stability could be improved by modifying the laminates parameter [4]. Some of these couplings, such as the bending torsion, may be present even in symmetrical laminates. Because of this latter coupling, beams subjected to transverse load may undergo not only bending moments but also torsional moments or rotations.

Bending torsion coupling can appear in a beam for two main reasons: due to the geometry of the section, when the shear centre

\footnotetext{
* Corresponding author. Tel.: +34916249965; fax: +34916249430.

E-mail address: ebarbero@ing.uc3m.es (E. Barbero).
}

does not coincide with the centroid [5,6], or due to the anisotropy of the material, as in the case of composite laminates. In the laminates, the bending torsion coupling can appear regardless of the geometry of the beam cross section, when there are laminas with orientations other than $0^{\circ}$ or $90^{\circ}$, even if the stacking sequence is symmetrical. This coupling is controlled by a stiffness parameter, which needs to be calculated from the elastic constants of the lamina [3,7]. Many authors have studied the bending torsion coupling of laminated beams, both in solid cross sections and in thin walled cross sections.

The study of laminated beams with thin walled cross sections has focussed on calculating natural frequencies or mode shapes, both in closed sections [2] and open ones [8], as well as on calculating displacements [7,9,10]. Dancila and Armanios [2] used an analytical model, considering the bending torsion and tensile torsion couplings in a closed section, and validated the results with a finite element model. Lee and Kim [8] used a one dimensional finite element model applicable to open thin walled cross sections. Chandra et al. [7] calculated static displacements of a carbon/epoxy beam with a box section, comparing experimental results with those found by a finite element model and an analytical model. Jung et al. [11] studied the bending torsion coupling effect in closed cross section beams, analysing their response to static loads by calculating of the bending and torsional stiffness as functions of the stacking sequence of the laminate. Li et al. [9] analysed the dynamic response of thin walled Timoshenko beams, considering concentrated and distributed dynamic loads, and bending torsion coupling.

Studies on solid cross section beams have dealt primarily with the analysis of vibrations of laminated cantilever beams. Banarjee 


\begin{tabular}{|c|c|c|}
\hline Nomenclature & $q_{2}(x, t)$ & applied bending moment per unit width of the beam \\
\hline & $q_{3}(x, t)$ & applied torsional moment per unit width of the beam \\
\hline $\begin{array}{l}123 \text { axis principal material coordinate system } \\
x y z \text { axis global beam coordinate system }\end{array}$ & {$[R]$} & $\begin{array}{l}\text { diagonal matrix depending on the integration algo } \\
\text { rithm }\end{array}$ \\
\hline$x^{\prime} y^{\prime} z^{\prime}$ axis global laminate coordinate system & {$\left[R^{*}\right]$} & diagonal matrix depending on the integration algo \\
\hline A beam cross sectional area & & rithm calculated in $n$ Gauss points \\
\hline extensional stiffness matrix & $t$ & time $(s)$ \\
\hline shear stiffness matrix & $t_{c}$ & beam first natural period of vibration calculated by \\
\hline shear flexibility matrix & & FEM3D \\
\hline beam width & $u, v, w$ & displacement components along $x, y, z$ coordinate \\
\hline flexibility influence functions matrix & & directions, respectively \\
\hline $\begin{array}{l}\text { flexibility influence functions matrix calculated in } n \\
\text { Gauss points }\end{array}$ & \multicolumn{2}{|c|}{$u_{0}, v_{0}, w_{0}$ displacement components of a point on the midplane } \\
\hline bending stiffness matrix & $\delta\left(\begin{array}{ll}x & \xi\end{array}\right)$ & Dirac Delta function \\
\hline bending flexibility matrix & $\vec{\delta}(x, t)$ & generalized displacement of a point over the time on a \\
\hline lamina longitudinal modulus in direction 1 & & beam \\
\hline lamina transverse modulus in direction 2 & $\vec{\delta}^{*}(t)$ & generalized displacement vector calculated in $n$ Gauss \\
\hline lamina transverse modulus in direction 3 & & points \\
\hline exterior loads vector & $\underset{\substack{\mathbb{\delta}_{s t} \\
*}}{ }(\chi, t)$ & generalized displacement vector in static conditions \\
\hline lamina shear modulus in plane 12 & $\delta_{s t}(t)$ & generalized displacement vector in static conditions \\
\hline lamina shear modulus in plane 13 & & calculated in $n$ Gauss points \\
\hline $\begin{array}{l}\text { lamina shear modulus in plane } 23 \\
\text { beam thickness }\end{array}$ & $\varepsilon_{x x}, \quad \varepsilon_{y y}$ & $\begin{array}{l}\varepsilon_{z z} \text { longitudinal strains in the } x, y, z \text { coordinate } \\
\text { directions, respectively }\end{array}$ \\
\hline $\begin{array}{l}\text { moment of inertia of the beam cross section about the } \\
y \text { axis }\end{array}$ & $\begin{array}{ll}\varepsilon_{x x}^{0}, & \varepsilon_{y y}^{0} \\
\varepsilon_{x x}^{1}, & \varepsilon_{y y}^{1}\end{array}$ & $\begin{array}{ll}\gamma_{x y}^{0} & \text { membrane strains } \\
\gamma_{x y}^{1} & \text { flexural strains (curvatures) }\end{array}$ \\
\hline $\begin{array}{l}\text { polar moment of inertia } \\
\text { shear correction factor }\end{array}$ & $\phi_{x}$ & $\begin{array}{l}\text { bending rotation (rotation of a transverse normal } \\
\text { about the } y \text { axis) }\end{array}$ \\
\hline support span of the beam & $\phi_{x}^{o}$ & bending rotation calculated by FEM3D under static \\
\hline generalized mass matrix calculated in $n$ Gauss points & $\phi_{y}$ & torsional rotation (rotation of a transverse normal \\
\hline$M_{x x}, M_{y y}$ bending moments per unit width & & about the $x$ axis) \\
\hline $\begin{array}{ll}M_{x y} & \text { torsional moments per unit width } \\
{[m(x)]} & \text { mass matrix }\end{array}$ & $\phi_{y}^{o}$ & $\begin{array}{l}\text { torsional rotation calculated by FEM3D under static } \\
\text { conditions }\end{array}$ \\
\hline$N_{x x}, N_{y y}$ in plane forces per unit width & $\gamma_{x z}, \gamma_{y z}$ & interlaminar shear strains \\
\hline in plane shear force per unit width & $\gamma_{x y}$ & in plane shear strain \\
\hline dynamic forces function & $v_{21}$ & major Poisson ratio in plane 12 \\
\hline$Q_{x y}, Q_{y y}$ interlaminar shear forces per unit width & $v_{31}$ & major Poisson ratio in plane 13 \\
\hline $\bar{Q}_{i j}^{(k)} \quad$ components of lamina stiffness matrix & $v_{32}$ & major Poisson ratio in plane 23 \\
\hline $\begin{array}{l}q_{1}(x, t) \text { transverse distributed force per unit width of the } \\
\text { beam }\end{array}$ & $\rho$ & material density \\
\hline
\end{tabular}

and Williams [12] determined the frequencies of solid section cantilever beams manufactured using unidirectional laminate with fibres oriented to $15^{\circ}$, without explaining the choice of this orientation. Later, Banarjee [13] completed that work by deter mining the exact expressions for frequencies and mode shapes of the same composite beam. Teh and Huang [14] evaluated the effect of the orientation of the fibres on the frequencies and mode shapes of cantilever graphite/epoxy beams, stating that the maximum coupling effect varies from $24^{\circ}$ to $25^{\circ}$ for the first five natural frequencies. Jun and Xianding [3] used modal analysis to study the flexural behaviour of composite beams, determining the displacement and flexure rotation of a cantilever beam subjected to concentrated and distributed random excitations.

In addition to considering the effect of bending torsion coupling to predict the dynamic response of a beam, it is necessary to include in the models the effect of shear deformation and rotary inertia. In isotropic beams, it is possible in some cases to disregard the effects of shear deformation and rotary inertia [1]. However, the effects of interlaminar shear stress need to be taken into account in the study of laminated beams due to their low interlaminar shear modulus [16]. High order shear theories have been developed [ $\left.\begin{array}{ll}17 & 20\end{array}\right]$ but usually the First Order Shear Deformation Laminate Theory is used, as it provides a result similar to that of higher order theories but with lower computa tional cost [21].

To analyse the behaviour of laminated beams, some authors use numerical techniques such as the Finite Element Method, the Galerkin Method or the Boundary Element Method [8,22 24], whereas others use analytical models $[3,13,15,25,26]$. The latter is useful in the optimisation processes because they offer a simple way to evaluate the influence of the different parameters in the overall response of the structure.

To solve the equations of motion of the beam by simplified methods, most researchers use Modal Analysis [3,12 14,27,28]. However, if the beam has a variable bending stiffness and the boundary conditions are hyperstatic, the equations of motion are complex to solve by Modal Analysis. Different methods have been used in this case, such as the Boundary Integral Equation Method [5], the Transfer Matrix Method [16], the Differential Quadrature Method [29], the Green Function [30], or the Flexibility Influence Function Method (FIFM) [31].

The FIFM is a technique that does not require the calculation of the natural frequencies or vibration modes of the beam [32]. This method is especially useful for solving hyperstatic problems in dynamic conditions because the influence of the boundary conditions in this method is restricted to the solution of equations 
for static conditions. After having solved the hyperstatic problem for static conditions, the dynamic equations can be solved by a single technique, independently of the boundary conditions. On the contrary, other methods (e.g. modal analysis) require different dynamic equations to be solved for each boundary condition. The FIFM was validated in a previous work [31] by comparisons with Modal Analysis and Finite Elements Method in the calculation of the deflection and bending rotation in composite beams with a stacking sequence of $0 / 90$. However, bending torsion coupling was not present in those beams.

Most studies deal with cantilever beams, as they are representative of many structural components with bending torsion coupling effect. However, these beams are isostatic and, due to bending torsion coupling, only a torsional rotation occurs. On the contrary, in a hyperstatic beam this coupling produces both torsional rotation and torsional moment, and, at the same time, this torsional moment produces deflection and bending rotation in the beam.

The present work analyses the behaviour of a bending torsional coupled laminate beam with hyperstatic boundary conditions, subjected to an impulsive load by an analytical model in which the equations were solved by FIFM. Both the deflection and torsional rotation were restricted at its ends, the bending rotation being totally free. Two analytical models were used: the first one includes the bending torsion coupling effect (FIFM) and the second one does not consider this coupling (FIFM non coupled). The deflection, bending rotation, and torsional rotation of this beam were calculated by the analytical models and compared with those determined by a FEM3D model.

\section{Derivation of equations of motion}

\subsection{Kinematics}

Considering bending torsional coupling effect and First order Shear Deformation Theory, the dynamic behaviour of a beam can be defined by calculating three variables: deflection, bending rotation, and torsional rotation.

In the First order Shear Deformation laminated plate Theory, two hypotheses are assumed: the straight lines perpendicular to the midsurface (i.e., transverse normals) remain straight after deformation and do not elongate. However, it is not assumed that transverse normals remain perpendicular to the midsurface after deformation. The displacement field is of the form [1]:

$$
\left\{\begin{array}{l}
u(x, y, z, t)=u_{0}(x, y, t)+z \phi_{x}(x, y, t) \\
v(x, y, z, t)=v_{0}(x, y, t)+z \phi_{y}(x, y, t) \\
w(x, y, z, t)=w_{0}(x, y, t)
\end{array}\right.
$$

By derivation of the displacement field, the following strains result:

$$
\left\{\begin{array}{c}
\varepsilon_{x x} \\
\varepsilon_{y y} \\
\varepsilon_{z z} \\
\gamma_{y z} \\
\gamma_{x z} \\
\gamma_{x y}
\end{array}\right\}=\left\{\begin{array}{c}
\frac{\partial u_{0}}{\partial x} \\
\frac{\partial v_{0}}{\partial y} \\
0 \\
\frac{\partial w_{0}}{\partial y}+\phi_{y} \\
\frac{\partial w_{0}}{\partial x}+\phi_{x} \\
\left(\frac{\partial u_{0}}{\partial y}+\frac{\partial v_{0}}{\partial x}\right)
\end{array}\right\}+z\left\{\begin{array}{c}
\frac{\partial \phi_{x}}{\partial x} \\
\frac{\partial \phi_{y}}{\partial y} \\
0 \\
0 \\
0 \\
\left(\frac{\partial \phi_{x}}{\partial y}+\frac{\partial \phi_{y}}{\partial x}\right)
\end{array}\right\}
$$

$\gamma_{x z}, \gamma_{y z}$ are constants through the thickness of the laminate and $\varepsilon_{z z}$ is zero.
The governing equations in this model have been derived using the dynamic version of the principle of virtual displacements. As a result the displacements can be expressed as a function of the forces as

$$
\begin{aligned}
& \frac{\partial N_{x x}}{\partial x}+\frac{\partial N_{x y}}{\partial y}=I_{0} \frac{\partial^{2} u_{0}}{\partial t^{2}}+I_{1} \frac{\partial^{2} \phi_{x}}{\partial t^{2}} \\
& \frac{\partial N_{x y}}{\partial x}+\frac{\partial N_{y y}}{\partial y}=I_{0} \frac{\partial^{2} v_{0}}{\partial t^{2}}+I_{1} \frac{\partial^{2} \phi_{y}}{\partial t^{2}} \\
& \frac{\partial Q_{x x}}{\partial x}+\frac{\partial Q_{y y}}{\partial y}+\frac{\partial}{\partial x}\left(N_{x x} \frac{\partial w_{0}}{\partial x}+N_{x y} \frac{\partial w_{0}}{\partial y}\right) \\
& \quad+\frac{\partial}{\partial y}\left(N_{x y} \frac{\partial w_{0}}{\partial x}+N_{y y} \frac{\partial w_{0}}{\partial y}\right)+q_{1}(x, t)=I_{0} \frac{\partial^{2} w_{0}}{\partial t^{2}} \\
& \frac{\partial M_{x x}}{\partial x}+\frac{\partial M_{x y}}{\partial y} \quad Q_{x x}+q_{2}(x, t)=I_{2} \frac{\partial^{2} \phi_{x}}{\partial t^{2}}+I_{1} \frac{\partial^{2} u_{0}}{\partial t^{2}} \\
& \frac{\partial M_{x y}}{\partial x}+\frac{\partial M_{y y}}{\partial y} \quad Q_{y y}+q_{3}(x, t)=I_{2} \frac{\partial^{2} \phi_{y}}{\partial t^{2}}+I_{1} \frac{\partial^{2} v_{0}}{\partial t^{2}}
\end{aligned}
$$

where

$I_{i}=\int_{-h / 2}^{h / 2} z^{i} \rho \mathrm{d} z, \quad i=0,1,2$

$I_{1}$ is zero for a symmetrical laminated beam.

\subsection{Constitutive equations}

Integration of the stresses through the thickness of laminate provides the constitutive equations, where the force and the moment resultants are related to the strains. In a symmetrical laminate the constitutive equations are of the form:

$\left\{\begin{array}{c}N_{x x} \\ N_{y y} \\ N_{x y}\end{array}\right\}=\left[\begin{array}{lll}A_{11} & A_{12} & A_{16} \\ A_{12} & A_{22} & A_{26} \\ A_{16} & A_{26} & A_{66}\end{array}\right]\left\{\begin{array}{c}\varepsilon_{x x}^{0} \\ \varepsilon_{y y}^{0} \\ \gamma_{x y}^{0}\end{array}\right\}$

$\left\{\begin{array}{l}M_{x x} \\ M_{y y} \\ M_{x y}\end{array}\right\}=\left[\begin{array}{lll}D_{11} & D_{12} & D_{16} \\ D_{12} & D_{22} & D_{26} \\ D_{16} & D_{26} & D_{66}\end{array}\right]\left\{\begin{array}{c}\varepsilon_{x x}^{1} \\ \varepsilon_{y y}^{1} \\ \gamma_{x y}^{1}\end{array}\right\}$

$\left\{\begin{array}{l}Q_{y y} \\ Q_{x x}\end{array}\right\}=K\left[\begin{array}{cc}A_{44}^{s} & A_{45}^{s} \\ A_{54}^{s} & A_{55}^{s}\end{array}\right]\left\{\begin{array}{l}\gamma_{y z} \\ \gamma_{x z}\end{array}\right\}$

For a general laminate, the shear correction factor $K$ depends on lamina properties and the stacking sequence. In this work, $K=5 / 6$ was used, as in a rectangular section of a homogeneous beam because the differences between this shear correction factor and specifics composite shear correction factors [33,34] are not im portant in the studied beam, as demonstrated in Santiuste [35].

The laminate stiffness matrices $\left([A],[D]\right.$ and $\left.\left[A^{s}\right]\right)$ are defined in terms of the components of the lamina stiffness matrix, $\bar{Q}_{i j}^{(k)}$, as

$$
\begin{aligned}
& A_{i j}=\sum_{k 1}^{N} \bar{Q}_{i j}^{(k)}\left(z_{k+1} \quad z_{k}\right) \quad i, j=1,2,6 \\
& D_{i j}=\frac{1}{3} \sum_{k}^{N} \bar{Q}_{i j}^{(k)}\left(z_{k+1}^{3} \quad z_{k}^{3}\right) \\
& A_{m n}^{s}=\sum_{k}^{N} \bar{Q}_{m n}^{(k)}\left(z_{k+1} \quad z_{k}\right) \quad m, n=4,5
\end{aligned}
$$

In the present study, a laminate beam subjected to transverse loads was studied. If a bending torsion coupled beam has hyperstatic boundary conditions, when it is subjected to trans verse loads, besides bending rotation and displacements, torsional rotations and torsional moments appear. 
When only $Q_{x x}, M_{x x}$ and $M_{x y}$ are considered:

$N_{x x}=N_{y y}=N_{x y}=0$

$M_{y y}=0$

$Q_{y y}=0$

Assuming that $\partial / \partial y=0$, the constitutive equations are reduced to

$$
\begin{aligned}
& \frac{\partial \phi_{x}}{\partial x}=d_{11} M_{x x}+d_{16} M_{x y} \\
& \frac{\partial w_{0}}{\partial x}+\phi_{x}=\frac{a_{55}^{s}}{K} Q_{x x} \\
& \frac{\partial \phi_{y}}{\partial x}=d_{16} M_{x x}+d_{66} M_{x y}
\end{aligned}
$$

where $a_{55}^{s}, d_{11}, d_{16}$ and $d_{66}$ are terms of the flexibility matrices $\left[a^{s}\right]$ and $[d] . a_{55}^{s}$ indicates the shear flexibility of the laminate, $d_{11}$ the bending flexibility, $d_{16}$ the bending torsion coupling flexibility, and $d_{66}$ the torsional flexibility. In a symmetrical laminate the matrices $\left[a^{s}\right]$ and $[d]$ are the inverses of $\left[A^{s}\right]$ and $[D]$, and it is possible to express the terms $a_{55}^{s}, d_{11}, d_{16}$, and $d_{66}$ as

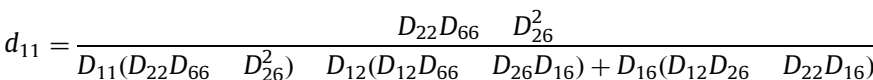

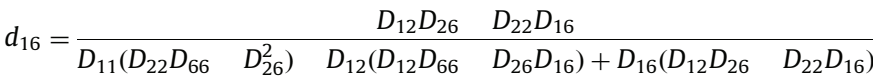

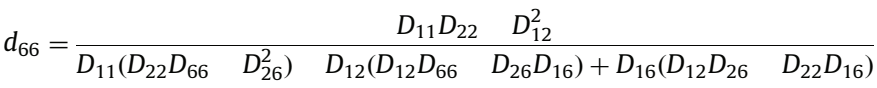
$a_{55}^{s}=\frac{A_{44}^{s}}{A_{44}^{s} A_{55}^{s} \quad A_{45}^{s^{2}}}$

Substituting strains and forces, assuming that $\partial / \partial y=0$, in terms of the displacements yield three second order coupled differential equations:

$\frac{K}{a_{55}^{s}}\left(\frac{\partial^{2} w_{0}}{\partial x^{2}}+\frac{\partial \phi_{x}}{\partial x}\right)+q_{1}(x, t)=I_{0} \frac{\partial^{2} w_{0}}{\partial t^{2}}$,

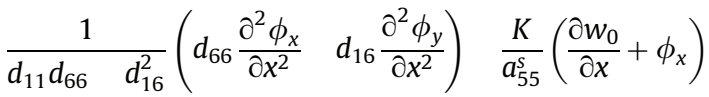

$+q_{2}(x, t)=I_{2} \frac{\partial^{2} \phi_{x}}{\partial t^{2}}$

$\frac{1}{d_{11} d_{66} d_{16}^{2}}\left(d_{16} \frac{\partial^{2} \phi_{x}}{\partial x^{2}}+d_{11} \frac{\partial^{2} \phi_{y}}{\partial x^{2}}\right)+q_{3}(x, t)=I_{2} \frac{\partial^{2} \phi_{y}}{\partial t^{2}}$

These equations must be solved according to the boundary and initial conditions. In this works it is assumed that the beam is at rest initially.

\subsection{Flexibility influence functions method}

The FIFM was used to solve Eq. (12), enabling the calculation of the displacements occurring in a one dimensional continuous system by the solution of an integral equation. The generalized displacement of a point over time, $\vec{\delta}(x, t)$, in a beam of length $L$ subjected to a generic dynamic force, $\vec{p}(\xi, t)$, can be calculated by the following expression:

$\vec{\delta}(x, t)=\int_{0}^{L}[C(x, \xi)] \vec{p}(\xi, t) \mathrm{d} \xi$

The displacements are expressed in a generalized displacement vector with three variables: deflection, bending rotation, and torsional rotation:

$\vec{\delta}(x, t)=\left(\begin{array}{c}w(x, t) \\ \phi_{x}(x, t) \\ \phi_{y}(x, t)\end{array}\right)$
The matrix $[C(x, \xi)]$ contains the influence functions:

$[C(x, \xi)]=\left(\begin{array}{lll}C^{w w}(x, \xi) & C^{w b}(x, \xi) & C^{w t}(x, \xi) \\ C^{b w}(x, \xi) & C^{b b}(x, \xi) & C^{b t}(x, \xi) \\ C^{t w}(x, \xi) & C^{t b}(x, \xi) & C^{t t}(x, \xi)\end{array}\right)$

In the influence functions matrix, each component has a physical meaning, for example the function $C^{w b}(x, \xi)$ is defined as the vertical deflection of a section located at distance $x$ from the left end due to a unit static bending moment applied at the point $\xi$ (Fig. 1). This function was calculated by integration of Eq. (10), considering the shear force, bending and torsional moments corresponding to

$q_{2}(x, t)=\delta(x \quad \xi)$

where $\delta(x \xi)$ is the Dirac Delta function.

The $C^{w b}(x, \xi)$ could be calculated as

$$
\begin{aligned}
C^{w b}(x, \xi)= & w_{o}^{s t}(0)+x \phi_{x}^{s t}(0)+\int_{0}^{x} d_{11} M_{x x}(s, \xi) s \mathrm{~d} s \\
& +\int_{0}^{x} d_{16} M_{x y}(s, \xi) s \mathrm{~d} s+\int_{0}^{x} a_{55} Q_{x x}(s, \xi) s \mathrm{~d} s
\end{aligned}
$$

In this equation, $M_{x x}(s, \xi), M_{x y}(s, \xi)$ and $Q_{x x}(s, \xi)$ are the bending moment, the torsional moment and shear force of the problem depicted in Fig. 1, while $w_{o}^{s t}(0)$ and $\phi_{x}^{\text {st }}(0)$ are the vertical deflection and bending rotation in the left support $(x=0)$.

The other elements of the matrix $[C(x, \xi)]$ can be calculated in a similar way.

The influence of the Boundary conditions in FIFM is restricted to the solution of equations under static conditions, as in Eq. (17). After having solved these static equations, the dynamic equations which are detailed as follows can be solved by a single technique, regardless of the boundary conditions. On the contrary, other methods (e.g. modal analysis) require different dynamic equations to be solved for each boundary condition.

In Eq. (13) the function $\vec{p}(\xi, t)$ contains the dynamic forces: exterior loads and inertia forces:

$\vec{p}(\xi, t)=[m(\xi)] \frac{\partial^{2} \vec{\delta}(\xi, t)}{\partial t^{2}}+\vec{f}(\xi, t)$
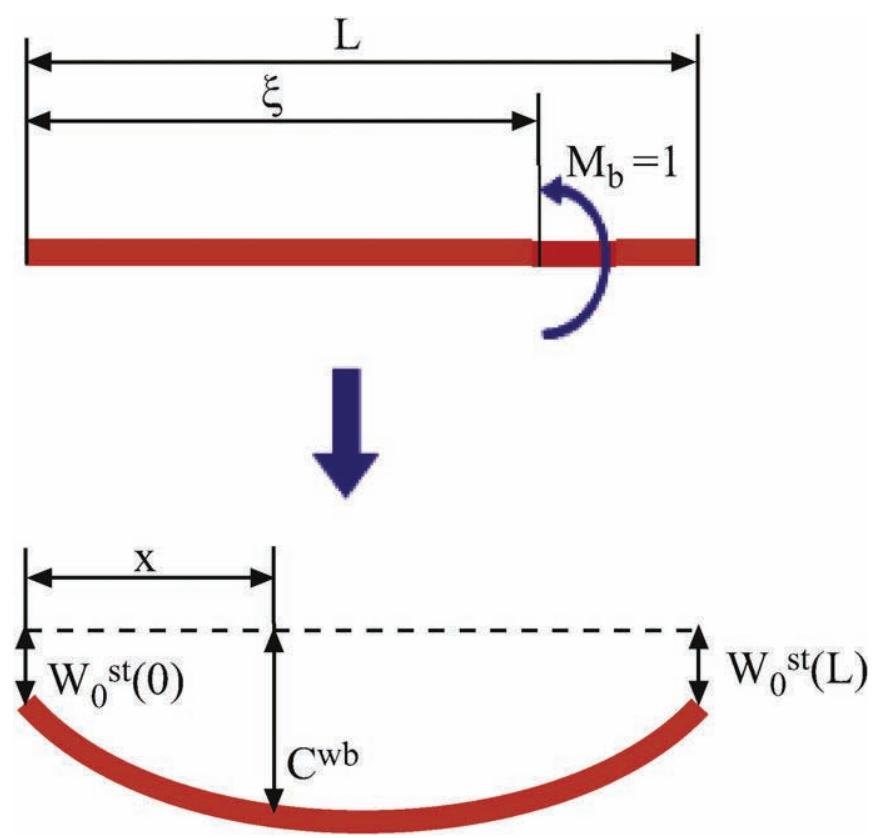

Fig. 1. Physical meaning of the $C^{w b}(x, \xi)$ function. 
where

$\vec{f}(\xi, t)=\left(\begin{array}{c}q_{1}(\xi, t) \\ q_{2}(\xi, t) \\ q_{3}(\xi, t)\end{array}\right) \quad[m(\xi)]=\left(\begin{array}{ccc}\rho A & 0 & 0 \\ 0 & \rho I_{y y} & 0 \\ 0 & 0 & \rho J\end{array}\right)$

Substituting in Eq. (13) yields

$\int_{0}^{L}[C(x, \xi)][m(\xi)] \frac{\partial^{2} \vec{\delta}(\xi, t)}{\partial t^{2}} \mathrm{~d} \xi+\vec{\delta}(x, t)=\vec{\delta}_{s t}(x, t)$

where

$\vec{\delta}_{s t}(x, t)=\int_{0}^{L}[C(x, \xi)] \vec{f}(\xi, t) \mathrm{d} \xi$

Eq. (20) was transformed into a system of second order differential equations by a numerical integration using the Gauss method:

$\left[C^{*}\right]\left[R^{*}\right]\left[M^{*}\right] \frac{\partial^{2} \vec{\delta}^{*}(t)}{\partial t^{2}}+\vec{\delta}^{*}(t)=\vec{\delta}_{s t}^{*}(t)$

where $\left[C^{*}\right],\left[R^{*}\right]$ and $\left[M^{*}\right]$ matrix sizes are $3 n \times 3 n$, while $\vec{\delta}^{*}(t)$ and $\vec{\delta}_{s t}^{*}(t)$ are vectors of $3 n$ components.

Eq. (22) can be transformed into

$\frac{\partial^{2} \vec{\delta}^{*}(t)}{\partial t^{2}}=\left(\left[C^{*}\right]\left[R^{*}\right]\left[M^{*}\right]\right)^{-1}\left(\vec{\delta}_{s t}^{*}(t) \quad \vec{\delta}^{*}(t)\right)$

The problem is reduced to solving a system of $3 n$ second order differential equations. In this work, the Stoerm rule [36] was used to solve the system. For this method to be applied, the first derivates of the unknown functions cannot appear in the equation.

\section{Analysis of bending-torsional coupling effect}

For the analysis of the dynamic behaviour of a laminate with bending torsion coupling, a beam with a point load at its midpoint was considered. The bending torsional coupling of laminates with different stacking sequences was studied, select ing the sequence that produces the maximum coupling. For that laminate the dynamic displacements of the beam were calculated by solving Eq. (23), considering the bending torsion coupling (FIFM) and they were compared with those determined when the coupling is not included in the equations of motion (the equations of the FIFM for the case without coupling are detailed in Santiuste et al. [31]). The accuracy of this method was assessed by comparing the displacements to those calculated by a three dimensional finite elements model (FEM3D).

\subsection{Material and geometry}

The geometry of the beam studied was: thickness $1.6 \mathrm{~mm}$, width $3.2 \mathrm{~mm}$ and support span $24 \mathrm{~mm}$. The selected span to thickness ratio, 15, was low enough for the transverse shear stresses to influence the behaviour of this orthotropic laminate [35]. The composite used was a carbon/epoxy laminate, for which the lamina elastic properties are shown in Table 1.

Table 1

Elastic properties of the lamina.

\begin{tabular}{lllllllll}
\hline$E_{1}(\mathrm{GPa})$ & $E_{2}(\mathrm{GPa})$ & $E_{3}(\mathrm{GPa})$ & $G_{12}(\mathrm{GPa})$ & $G_{13}(\mathrm{GPa})$ & $G_{23}(\mathrm{GPa})$ & $v_{21}$ & $v_{31}$ & $v_{32}$ \\
\hline 114 & 10 & 10 & 6.2 & 6.2 & 6.2 & 0.28 & 0.4 & 0.4 \\
\hline
\end{tabular}

\subsection{Stacking sequence}

The bending torsion coupling was studied in three laminates with different stacking sequences: laminate $[0]_{8}$, laminate $[0 / 90]_{2 \mathrm{~s}}$ and laminate $[30 / 30]_{2 S}$. For each laminate, the variation of the coupling was analysed, calculating the $d_{16}$ term when the laminate rotation angle $(\theta)$ rotates from $0^{\circ}$ to $180^{\circ}$. The laminate rotation angle $(\theta)$ is the angle formed between the longitudinal direction of the beam ( $x$ axis) and the $x^{\prime}$ axis of the laminate.

In Fig. 2, the variation of the bending torsional coupling flexibility $\left(d_{16}\right)$ versus the laminate rotation angle $(\theta)$ was shown for the three laminates studied. In laminate $[0]_{8}$ the maximum coupling effect was greater than in the other laminates, while in laminate $[0 / 90]_{2 s}$, the maximum coupling was the lowest because the coupling caused by some plies is opposite to that caused by the perpendicular plies. Laminate $[30 / 30]_{2 s}$ presented an intermediate behaviour, as the sign of the coupling of all the plies could have been the same, but all the plies were never in the position of maximum coupling. In Fig. 2, it can also be seen that in laminates $[0 / 90]_{2 S}$ and $[30 / 30]_{2 S}$ there are rotation angles for which the factor $d_{16}$ is nil, and thus no bending torsion coupling exists in these cases. The greatest coupling occurs in laminate $[0]_{8}$, for which the $x^{\prime}$ axis forms an angle of $49.48^{\circ}$ with the longitudinal direction of the beam ( $x$ axis). Therefore, a laminate with a stacking sequence of $[50]_{8}$ was selected to analyse its dynamic behaviour by the FIFM and FEM3D.

\subsection{Boundary conditions and load}

The boundary conditions and the applied load were selected to reproduce the conditions of a three point bending test (Fig. 4). In this type of tests the beam is supported on two cylinders so that the bending rotation is completely free, while the displacement and torsional rotation in both supports are restricted. As the torsional rotation is restricted, a torsional moment of reaction appears, producing a torsional rotation and a bending rotation due to coupling in the beam. In the FIFM these boundary conditions are represented as

$x=0\left\{\begin{array}{l}w_{o}(0, t)=0 \\ \phi_{y}(0, t)=0 \\ \frac{\partial \phi_{x}(0, t)}{\partial x}=0\end{array} \quad x=L\left\{\begin{array}{l}w_{o}(L, t)=0 \\ \phi_{y}(L, t)=0 \\ \frac{\partial \phi_{x}(L, t)}{\partial x}=0\end{array}\right.\right.$

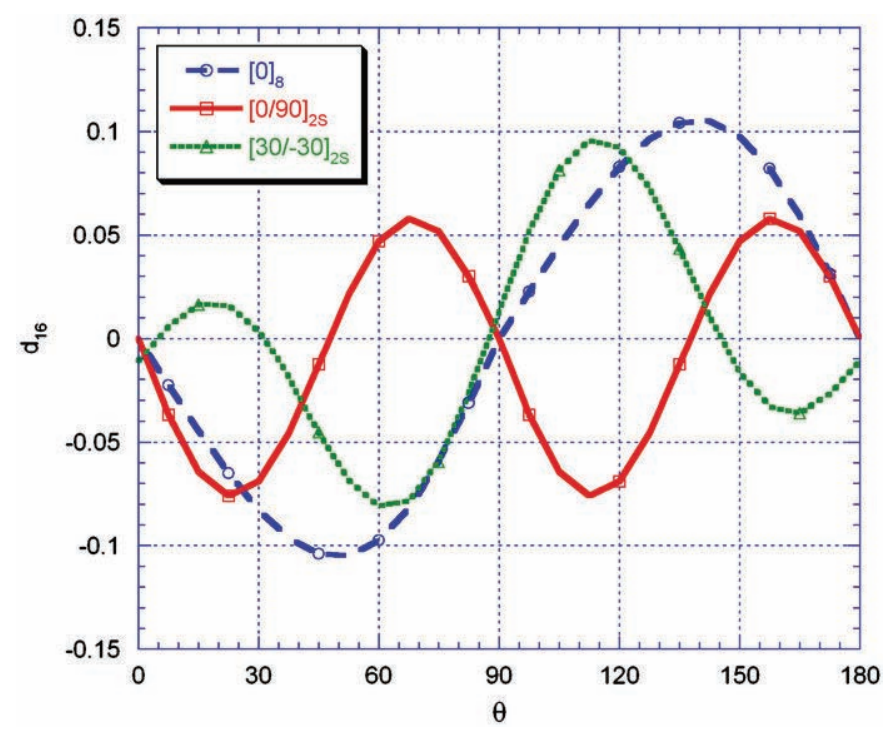

Fig. 2. Bending-torsion coupling flexibility versus ply rotation. 


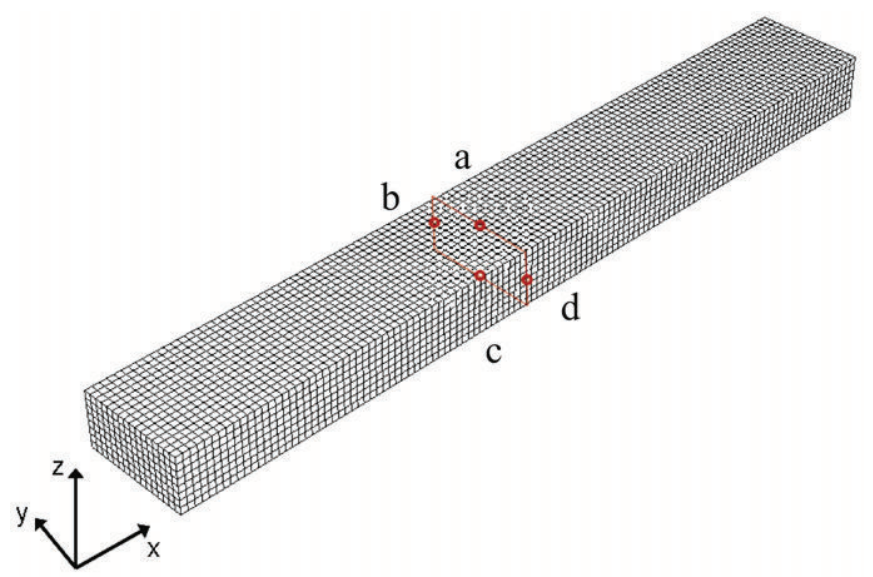

Fig. 3. FEM3D model mesh.

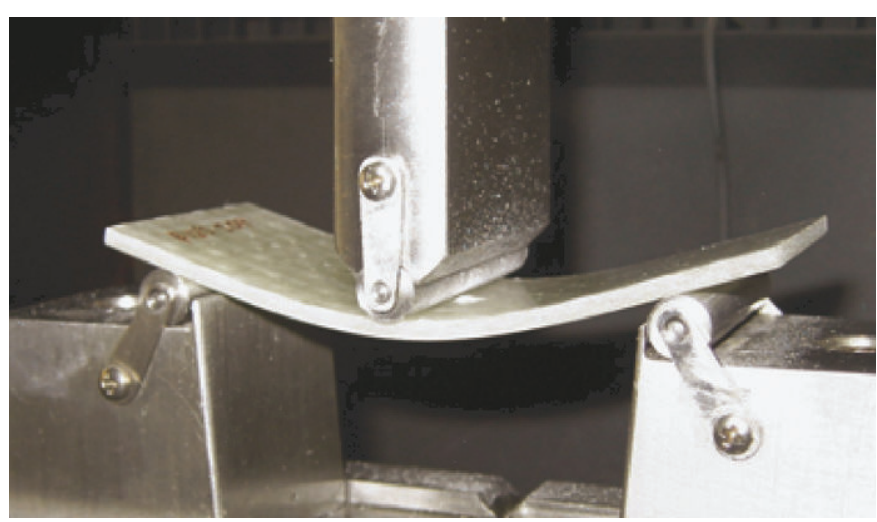

Fig. 4. Experimental three-points bending test configuration.

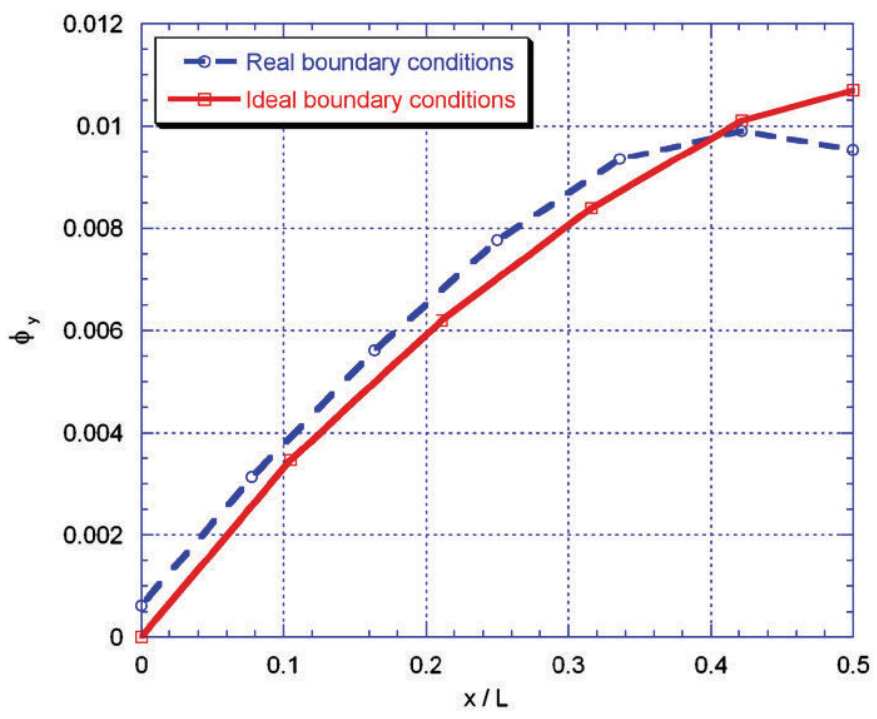

Fig. 5. Torsional rotation along longitudinal coordinate.

Two three dimensional numerical simulations were made to verify that the boundary conditions represented by Eq. (24) were similar to the experimental ones. In the first simulation, the boundary conditions shown in Fig. 4 were modelled, including the contact surfaces with the impactor and the supports. In the second, the problem was simplified with the boundary conditions used in the FIFM, Eq. (24) and the force was applied along the middle cross section of the test beam. Fig. 5 shows the torsional rotation caused in both models over the longitudinal coordinate from one of the supports to the central section.

The two results are quite similar, although two differences are evident. First, the point of contact with the supports, under real conditions cause a minor torsional rotation which is restricted under ideal conditions; that is, in the three point bending test the torsional rotation is not completely restricted at the supports. The second difference concerns the contact point with the impactor, $x / L=0.5$, which under real conditions causes an abrupt change in slope in the contact zone of the percutor, because this contact impedes to a certain degree the torsional rotation. As the dif ferences are minor, the boundary conditions used in the simplified model are considered valid.

An impulsive load (Eq. (25)) was applied on the beam in the middle cross section. The load time was selected to be equal to the first natural period of vibration of the beam, $t_{c}=259 \mu \mathrm{s}$, calculated by FEM3D. The maximum load applied, $F_{0}$, has a unitary value:

$F(t)=F_{0} \sin ^{3 / 2}\left(\pi \frac{t}{t_{C}}\right)$

\subsection{Numerical model}

A FEM3D model implemented in ABAQUS/Explicit [37] was used to verify the accuracy of the FIFM. The mesh was formed by 16000 elements which were eight nodes linear reduced integra tion brick elements (Fig. 3). The beam is composed of eight plies in which the elastic properties of the lamina were applied according to each ply orientation and the boundary conditions were applied at their ends. In the numerical model, the beam deflection was estimated as the vertical displacement of the centre of mass of the middle cross section.

In the FEM3D, the cross section did not remain plane after deformation, and therefore it was not possible to define a section rotation. For this reason, the bending and torsional rotations were calculated as an average rotation, as shown in Eq. (26), estimated by the displacements of two points (Fig. 3):

$\phi_{x}(x, t)=\frac{u_{a}(x, t) \quad u_{c}(x, t)}{h}$
$\phi_{y}(x, t)=\frac{w_{b}(x, t) \quad w_{d}(x, t)}{b}$

\section{Results}

To ascertain the dynamic response of the beam subjected to a load in its middle cross section, the deflection, bending rotation, and torsional rotation were calculated with the FIFM model at different points along the beam. With these values, a three dimensional representation of the beam was prepared at the moment of maximum deformation and this was compared with the image provided by the FEM3D model (Fig. 6). Qualitatively, the response by the analytical model is similar to that found with the numerical model.

In order to assess the accuracy of the analytical model (FIFM), the deflection, the bending and torsional rotations, were calcu lated and compared to those found by the three dimensional finite elements model (FEM3D). These values have been deter mined in a section situated at a quarter of the length of the beam, a section sufficiently far from the application point of the load and of supports to avoid effects of local compression in the results of the three dimensional numerical model. In the determination of the effect of the bending torsion coupling in the displacements 
calculated, they were also calculated by an analytical model in which this coupling was not considered (FIFM non coupled).

Fig. 7 shows the dynamic deflection, bending rotation, and torsional rotation divided by the corresponding static results determined with the FEM3D.

a

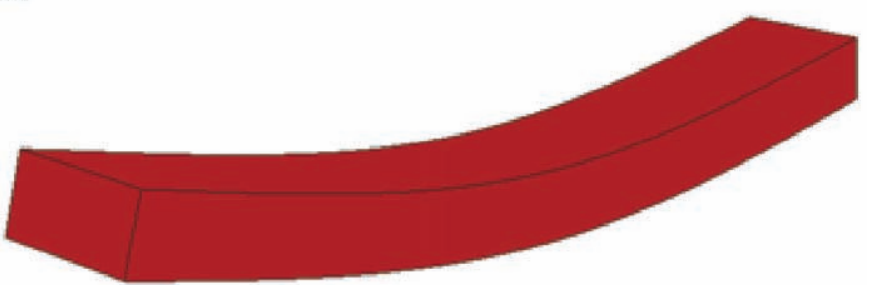

b

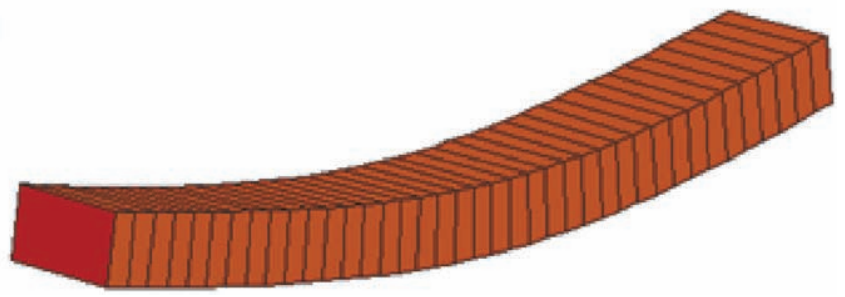

Fig. 6. Three-dimensional representation of the beam during maximum deformation: (a) FEM3D and (b) FIFM.
The results found with both models (analytical and numerical) varied in a similar way over time, being practically the same when the bending torsion coupling in the FIFM model was considered.

Table 2 shows the differences in the maximum deflection and rotations (bending and torsional) calculated by FIFM, FIFM non coupled and FEM3D. The differences in the maximum deflection and bending rotation calculated by FEM3D and FIFM were less than $7.5 \%$ and decreased significantly when the bending torsion coupling was taken into account in the analytical model, being lower than $1.25 \%$.

Fig. 7c shows that the FIFM can reproduce the variation of the torsional rotation with time and that the differences between the maximum results do not exceed 6\%. This difference is slightly greater than the deflection and bending rotation differences because the one dimensional model does not consider the warping caused in a rectangular cross section subjected to torsion, which is considered by the numerical model.

Table 2

Differences between the maximum deflection, bending rotation, and torsional rotation, calculated by FIFM and FEM3D.

\begin{tabular}{llll}
\hline & Deflection (\%) & Bending rotation (\%) & Torsional rotation (\%) \\
\hline FIFM (non-coupled) & 4.71 & 7.27 & - \\
FIFM & 1.17 & 1.21 & 5.81 \\
\hline
\end{tabular}

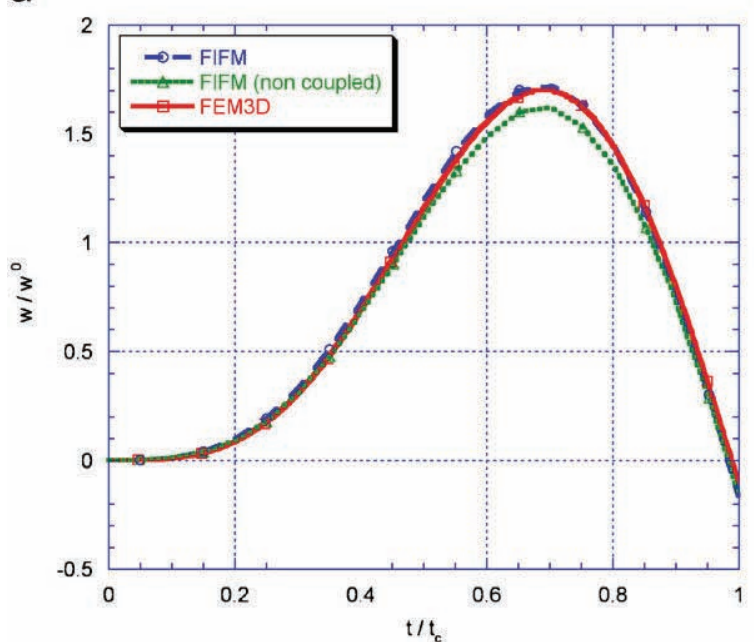

b

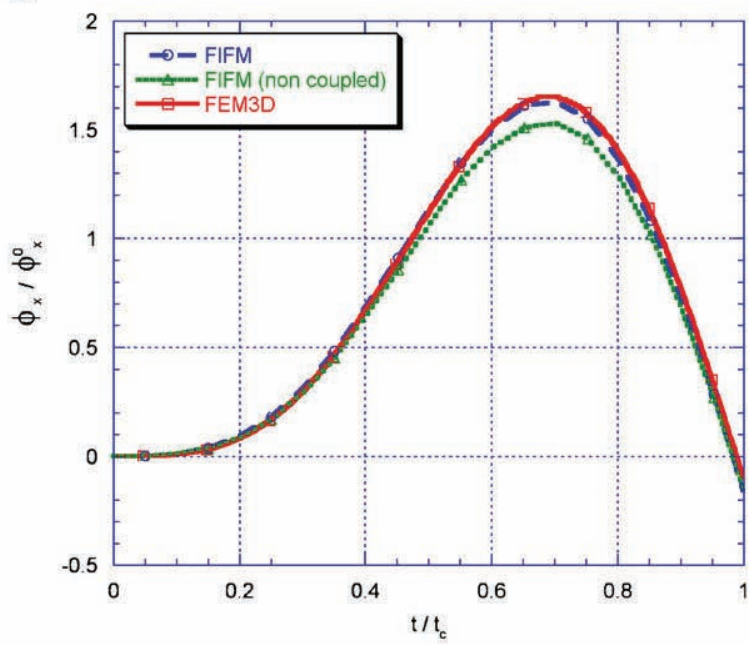

C

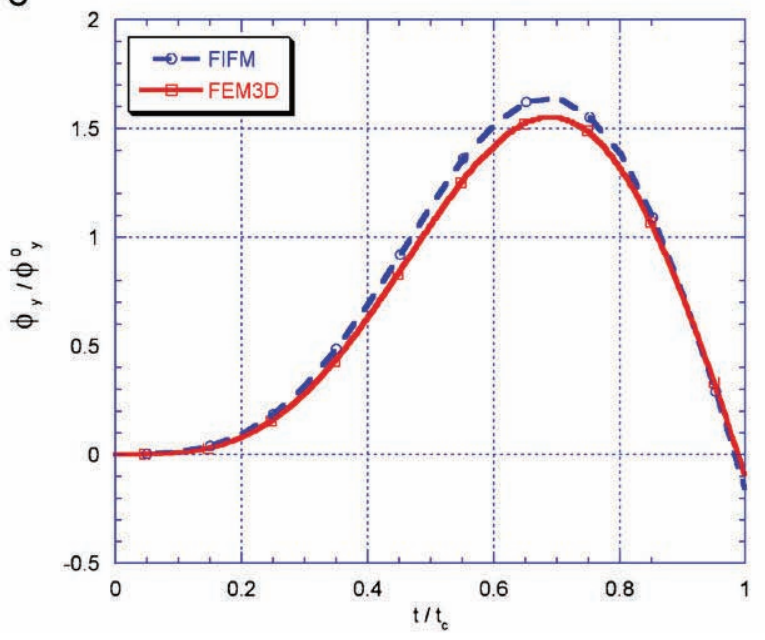

Fig. 7. Displacements in the section situated at a quarter of the length of the beam: (a) deflection, (b) bending rotation and (c) torsional rotation. 
Despite the simplification implicit in the one dimensional model, the FIFM model succeeds in calculating the deflection as well as bending and torsional rotations of a laminated beam. The accuracy of FIFM model is reasonable and the computational cost is lower than for the FEM3D model.

\section{Conclusions}

In this study, the dynamic behaviour of rectangular cross section laminated beams has been analysed considering the bending torsion coupling effect. An analytical model was em ployed while using the Flexibility Influence Functions Method to solve the equations of motion, and the results were compared with those provided by a three dimensional numerical model.

The main conclusions reached are the following:

- The bending torsion coupling effect needs to be considered in a one dimensional model in order to calculate the deflections and rotations of a laminated beam, because this coupling can have considerable effect on laminates with lamina orientations other than $0^{\circ}$ and $90^{\circ}$.

- In beams with hyperstatic boundary conditions, the bend ing torsion coupling effect influences the calculation of deflection and bending rotation as well as torsional rotation.

- The Flexibility Influence Functions Method is capable of solving the equations of motion of a beam, considering bending torsion coupling and interlaminar shear stress, with a lower computational cost than numerical methods and with a reasonable precision.

\section{References}

[1] Reddy JN. Mechanics of laminated composite plates: theory and analysis. FL: CRC Press; 1997.

[2] Dancila DS, Armanios EA. The influence of coupling on the free vibration of anisotropic thin-walled closed-section beams. International Journal of Solids and Structures 1998;35(23):3105-19.

[3] Jun L, Xianding J. Response of flexure-torsion coupled composite thin-walled beams with closed cross-sections to random loads. Mechanics Research Communications 2005;32(1):25-41.

[4] Rand O. A multilevel analysis of solid laminated composite beams International Journal of Solids and Structures 2001;38(22-23):4017-43.

[5] Fuller MJ, Holland PV, Tanaka M, Bercin AN, Suzuki R. Application of the boundary integral equation method to the coupled bending-torsional vibrations of elastic beams. Engineering Analysis with Boundary Elements 1997;20(1):73-9.

[6] Hashemi SM, Richard MJ. A dynamic finite element method for free vibration of bending-torsion coupled beams. Aerospace Science and Technology 2000;4(1):41-55.

[7] Chandra R, Stemple AD, Chopra I. Thin-walled composite beams under bending, torsional, and extensional loads. Journal of Aircraft 1990;27(7): 619-26.

[8] Lee J, Kim SE. Flexural-torsional coupled vibration of thin-walled composite beams with channel sections. Computers and Structures 2002;80(2):133-44.

[9] Li J, Shen R, Hua H, Jin X. Bending-torsional coupled dynamic response of axially loaded composite Timoshenko thin-walled beam with closed crosssection. Composite Structures 2004;64(1):23-35.
[10] Kim NI, Shin DK, Kim MY. Improved flexural-torsional stability analysis of thin-walled composite beam and exact stiffness matrix. International Journal of Mechanical Science 2007;49(1):950-69.

[11] Jung SN, Park I-J, Shin ES. Theory of thin-walled composite beams with single and double-cell sections. Composites Part B: Engineering 2007;38(2):182-92.

[12] Banerjee JR, Williams FW. Exact dynamic stiffness matrix for composite Timoshenko beams with applications. Journal of Sound and Vibration 1996; 194(4):573-85.

[13] Banerjee JR. Explicit analytical expressions for frequency equation and mode shapes of composite beams. International Journal of Solids and Structures 2001;38(14):2415-26.

[14] Teh KK, Huang CC. The effects of fibre orientation on free vibrations of composite beams. Journal of Sound and Vibration 1980;69(2):327-37.

[15] Li J, Wu G, Shen R, Hua H. Stochastic bending-torsion coupled response of axially loaded slender composite-thin-walled beams with closed crosssection. International Journal of Mechanical Science 2005;47(1):133-55.

[16] Yildirim V, Kiral E. Investigation of the rotary inertia and shear deformation effects on the out-of-plane bending and torsional natural frequencies of laminated beams. Composite Structures 2000;49(3):313-20.

[17] Levinson M. An accurate, simple theory of the statics and dynamics of elastic plates. Mechanics Research Communications 1980;7(6):343-50.

[18] Murthy MVV. An improved transverse shear-deformation theory for laminated anisotropic plates. NASA technical paper-1903; 1981.

[19] Reddy JN. A simple higher-order theory for laminated composite plates. Journal of Applied Mechanics 1984;51(4):745-52.

[20] Rand O. Interlaminar shear stresses in solid composite beams using a complete out-of-plane shear deformation model. Computers \& Structures 1998;66(6):713-23.

[21] Kapania RK, Raciti S. Recent advances in analysis of laminated beams and plates.1. Shear effects and buckling. AIAA Journal 1989;27(7):923-34.

22] Subrahmanyam KB, Kaza KRV, Brown GV, Lawrence C. Nonlinear vibration and stability of rotating, pretwisted, preconed blades including coriolis effects. Journal of Aircraft 1987;24(5):342-52.

[23] Khalid YA, Ali FA, Sahari BB, Saad EMA. Performance of composite I-beams under axial compression and bending load modes. Materials \& Design 2005;26(2):127-35.

[24] Mokos VG Sapountzakis EJ. A BEM solution to transverse shear loading of composite beams. International Journal of Solids and Structures 2005; 42(11-12):3261-87.

[25] Librescu L, Qin Z, Ambur DR. Implications of warping restraint on statics and dynamics of elastically tailored thin-walled composite beams. International Journal of Mechanical Science 2003:45(1):1247-67.

[26] Parlapalli MR, Shu D. Buckling of composite beams with two non-overlapping delaminations: Lower and upper bounds. International Journal of Mechanical Science 2007;49(1):793-805.

[27] Miller AK, Adams DF. An analytical means of determining the flexural and torsional resonant frequencies of generally orthotropic beams. Journal of Sound and Vibration 1975;41(4):433-49.

[28] Singh KV, Li G, Pang GS. Free vibration and physical parameter identification of non-uniform composite beams. International Journal of Solids and Structures 2006;74(1):37-50.

[29] Kuang J-H, Hsu M-H. The effect of fiber angle on the natural frequencies of orthotropic composite pre-twisted blades. Composite Structures 2002;58(4): 457-68.

[30] Hwu C. Green's function for the composite laminates with bending extension coupling. Composite Structures 2004;63(3-4):283-92.

[31] Santiuste C, Sánchez-Sáez S, Barbero E. Application of the flexibility influence function method in the dynamic analysis of composite beams. International Journal of Solids and Structures 2007;44(14-15):4795-809.

[32] Meirovitch L. Analytical methods in vibrations. New York: MacMillan Publishing Co.; 1967.

[33] Vinson JR, Sierakowski RL. The behaviour of structures composed of composite materials. Springer; 1986.

[34] Madabhusi-Raman P, Davalos JF. Static shear correction factor for laminated rectangular beams. Composites Part B: Engineering 1996;27(3-4):285-93.

[35] Santiuste C. Análisis y modelización de vigas de tipo laminado sometidas a cargas impulsivas. PhD thesis, Carlos III University of Madrid, 2007 [in Spanish].

[36] Press WH. FORTRAN numerical recipes. Cambridge: Cambridge University Press; 1994.

[37] HKS. Abaqus explicit v6.4 user's manual. Abaqus Inc.; 2003. 